Quantum physics

\section{How to make a}

\section{Schrödinger's cat}

Phys. Rev. Lett. 91, 130401 (2003)

Can a macroscopic object be placed in a quantum superposition - like Schrödinger's notorious cat, both dead and alive? William Marshall et al. have devised a scheme that comes close. Their 'cat' is not alive, nor exactly macroscopic; but it is far bigger than the atomic-scale entities with which superpositions are generally associated.

The researchers claim that a microscopic mirror, $10 \mu \mathrm{m}$ square and weighing around $5 \times 10^{-12} \mathrm{~kg}$, could be placed in a superposition such that it can be considered to occupy two positions simultaneously. The mirror is attached to a cantilever arm of the sort used in scanning probe microscopes, and forms one half of an optical cavity in which photons bounce back and forth. By coupling this cavity to an interferometer in which a light beam is split along two paths, a single photon placed in a superposition in the interferometer creates a superposition of the mirror. This would be revealed by the disappearance and reappearance of interference as the mirror cycles between its initial state and a quantum superposition.

Marshall et al. estimate that, for a mirror containing around $10^{14}$ atoms, the apparatus can be made sufficiently sensitive with existing technology. It would have to be cooled to around $2 \mathrm{mK}$, and the mirror itself further cooled using laser cooling to initialize it in the ground state. The technical demands are challenging, but seem feasible.

Philip Ball

\section{Neuroscience}

\section{Transport delays}

Neuron 40, 41-52 (2003)

Huntington's disease is an incurable, degenerative brain disorder. Sufferers carry an abnormal huntingtin protein, which contains longer repetitive stretches of the amino acid glutamine than normal. The protein is found inside nerve cells, often suspiciously close to vesicles - miniature suitcases that transport molecules along nerve-cell axons.

Györgyi Szebenyi et al. hypothesized that the mutant protein might disrupt this transport system. The team treated axons from giant squids with glutamine-rich fragments of the huntingtin protein. Vesicular transport slowed by more than $\mathbf{5 0} \%$. The same treatment also stopped intact human nerve cells from extending axons normally.

In the brain, these failures may prevent essential molecules from reaching their destination. As a result, neurons may malfunction and die, the authors speculate. Similar mechanisms may operate in other polyglutamine diseases, such as Kennedy's disease or spinobulbar muscular atrophy. Drugs that facilitate axonal transport may help to slow or block neurodegenerative diseases of this type.

Helen R. Pilcher

\section{Mineralogy}

\section{Oresome microbes}

Geology 31, 913-916 (2003)

Bacteria created massive zinc-ore deposits in Nevada, according to Thomas M. Bawden et $a l$. They have found what seems to be the first instance of 'supergene' formation of sphalerite (zinc sulphide, the principal commercial form of zinc ore). Supergene deposits are formed by the chemical or biological enrichment of an ore in a nearsurface environment. Previously, it was thought that supergene enrichment of sphalerite was thermodynamically unfavourable and unlikely to supply deposits suitable for mining.

Bacteria have a well-known capacity to induce mineral formation, but it is rare for this to happen on a scale big enough to produce economically viable mineral deposits. In the gold-rich strata studied by Bawden and colleagues - the Mike deposit in northern Nevada, which dates to the late Eocene (about 35 million years ago) sulphate-reducing bacteria apparently generated more than 400,000 tonnes of zinc ore during the subsequent Miocene epoch (24-25 million years ago). The involvement of bacteria is revealed by depletion of ${ }^{34} \mathrm{~S}$ in the sphalerite, a characteristic signature of a biogenic origin.

Philip Ball

\section{Planetary science Less of an impact Geology 31, 869-872 (2003)}

Meteorite impacts do not cause volcanic eruptions, according to B. A. Ivanov and H. J. Melosh. Their views run contrary to a theory popular among geologists, but Ivanov and Melosh say the theory doesn't hold water (or lava), and they have the calculations to support their case.

Since the first glimpse of lava-filled craters on the near side of the Moon in the 1960s, scientists have speculated that volcanism can be caused by decompression melting of crustal rock deep beneath impact craters. The lunar lava proved to be much younger than the impacts, but the idea persisted.
Using numerical simulations, Ivanov and Melosh calculated that a crater would need to be at least $500 \mathrm{~km}$ in diameter, twice the size of any known crater on Earth, before any molten material would reach the surface, even in relatively hot oceanic crust. Earth is thought to experience an impact of this size only once or twice every billion years. And an impact on land would have to be at least $1,200 \mathrm{~km}$ across. Even colossal impacts on other planets, such as the one that created the $2,000-\mathrm{km}$ Hellas Basin on Mars (pictured), failed to trigger volcanism.

Betsy Mason

\section{Genetics}

\section{Dyslexic inheritance}

Proc. Natl Acad. Sci. USA 100, 11553-11558 (2003)

Mikko Taipale and co-workers have pin-pointed a gene that, when disrupted, is implicated in dyslexia. It is normally found on the long arm of chromosome 15 , and is called DYX1C1. The protein it encodes is predicted to be 420 amino acids long; its function is unknown.

The starting point for Taipale et al. was a family in which the father and two daughters all suffered from dyslexia. It turned out that all three carried a translocation of DYX1C1 between chromosome 15 and chromosome 2. Further investigation of the gene in other groups of people with and without dyslexia revealed two changes in gene sequence that were associated with the disorder to greater or lesser extents.

The next steps will be to find out whether disrupted DYX1C1 occurs in broader samples of dyslexic patients, and whether it is involved in a more general learning disorder. Overall, the genetics of predisposition to dyslexia is likely to be fiendishly complex many variants of many genes might be involved, and some may be specific to different languages.

Tim Lincoln
Hell of a hit — the Hellas Basin on Mars. 